\title{
Survey Paper on Automatic Detection of Fake Profile Using Machine Learning on Instagram
}

\author{
Er. Pranay Meshram¹, Rutika Bhambulkar², Puja Pokale², Komal Kharbikar², Anushree Awachat ${ }^{2}$ \\ ${ }^{1}$ Assistant Professor, Department of Computer Science and Engineering, Priyadarshini J. L. College of \\ Engineering, Nagpur, Maharashtra, India \\ ${ }^{2}$ BE Scholar, Department of Computer Science and Engineering, Priyadarshini J. L. College of Engineering, \\ Nagpur, Maharashtra, India
}

\begin{abstract}
Article Info

Volume 8, Issue 3

Page Number: 46-50

Publication Issue :

May-June-2021

\section{Article History}

Accepted : 07 May 2021

Published: 13 May 2021

With the arrival of the Internet and social media, at the same time as masses of humans have benefitted from the full-size reassets of records available, there was an full-size boom with inside the upward push of cyber-crimes, mainly targeted closer to women. According to a 2019 file with inside the Economics Times, India has witnessed a $457 \%$ upward push in cybercrime with inside the 5 years span among 2011 and 2016. Most speculate that that is because of effect of social media inclusive of Facebook, Instagram and Twitter on our day by day lives. While those simply assist in growing a legitimate social network, advent of consumer debts in those websites normally desires simply an email-id. A actual lifestyles man or woman can create more than one fake IDs and for this reason impostors can effortlessly be made. Unlike the actual international state of affairs in which more than one policies and guidelines are imposed to become aware of oneself in a completely unique manner (as an instance at the same time as issuing one's passport or driver's license), with inside the digital international of social media, admission does now no longer require this kind of checks. In this paper, we study the one-of-a-kind debts of Instagram, specifically and try and verify an account as fake or actual the use of Machine Learning strategies specifically Logistic Regression and Random Forest Algorithm.
\end{abstract}

Keywords : Fakeprofile, Detection, Machine Learning, Social Media, Instagram, Internet

\section{INTRODUCTION}

In latest years, on line social networks (OSNs), which include Instagram, Twitter, and Facebook, have end up famous structures to disseminate and percentage information [1]. These offerings offer rapid and appropriate conversation and different forms of gear that make their customers be capable of without delay percentage and put up their multimedia contents which include pictures, movies, and audios over the 
internet [2]. Hence, except the huge range of customers on social structures, those capabilities and gear have involved many cyber criminals in the use of them to perform their malicious sports on social media structures efficiently. Unlike with inside the beyond, many assaults with a confined or small impact can now have a substantial effect via way of means of the use of on line social structures [3]. However, social medias impact is huge in peoples lives, and plenty of human beings use them to construct extra extensive connections [4]. One of the maximum famous social media is Instagram [5]. Instagram is a loose social networking app built for sharing images and movies over the internet. It is comparable to maximum different social media, wherein folks who create an account have a profile and information feed and may percentage images and movies via that.

In latest years, many celebrities and groups have created their debts on Instagram; they use Instagram to develop their commercial enterprise and fans [6]. Furthermore, a lot of them and different well-known customers use it as a platform for marketing and marketing. When a person is boosting the range of fans over a hundred thousand or millions, it's far no wonder to apply that humans account as a profitable earner. In the final years, many celebrities and everyday folks who reached a substantial range of fans on Instagram have used their debts as an area for marketing and marketing. People additionally attempt to growth the range in their fans for different reasons, which include attaining extra fame, being honest, and being influential. Such versatility and unfold of use have made Instagram the best platform for the proliferation of ordinary debts, which behave in uncommon ways. Most instructional researchers have basically targeted on spammers and debts, which put their efforts into spreading marketing and marketing, junk mail, malware, and different suspicious sports [7].
These malicious debts are generally the use of computerized applications to enhance their performance, conceal their actual identity, and appear to be actual customers. In beyond years, media have stated that account of celebrities, politicians, and a few famous commercial enterprise has indicated suspicious inflation of fans. Fake Instagram debts particularly used to growth the range of fans of a goal account. Therefore, artificially inflating the range of fans can additionally be concluded to acquire an account extra influential and honest with a view to stand from the gang to gain and entire extra valid fans to their account [8]. In beyond years, a number of the banks and monetary establishments with inside the U.S. determined to investigate social media debts of the mortgage applicants, earlier than sincerely giving the mortgage.

Hence, having a famous account can assist efficiently to growth the creditworthinessand reliability of the applicant. Furthermore, if a spammer followed fake fans, it could efficiently act as a valid person and put up extra authoritative messages and release diverse green marketing and marketing campaigns [9]. Some expert customers assume fake account detection is an smooth undertaking with their policies primarily based totally on anomaly account behaviour. Though, such policies are generally matched neither with analytic algorithms to combination them nor with validation mechanisms. Most instructional researchers have targeted in particular on junk mail and bot detection in diverse social media like Facebook and Twitter, with incredible outcomes in classifying fake debts primarily based totally on their valid and fraudulent features, mainly making use of machine-mastering strategies.

However, the papers last element proceeds as follows: the second one segment will deliver a short evaluate of the preceding studies in fake and spam debts detection in extraordinary environments and highlights their shortcomings and achievements.The 
$1 / 3$ segment describes the technique used for this study, along with the function extraction, and dataset. The fourth segment presents statistics approximately the proposed detection model, along with the used strategies and detection process. The 5th segment presents experiments along with an outline of the test setup, conditions, and assessment metrics used with inside the test. It additionally discusses the outcomes and the studies findings, besides evaluating the outcomes with different techniques. Finally, the sixth segment sums up the paper with concluding remarks.

\section{LITERATURE SURVEY}

1.In this paper creator proposed that the Social Networking is the primary technology of information transmission in addition to information introduction in a large scale and the motive why massive information is created is very lots known.[1] Social networking is the primary platform wherein lots of information is being created and via way of means of 2025 even Google information centres can't deal with that type of big extent. Increase with inside the fake money owed are developing exponential boom of the extent and on this paper we're providing and standing on their social networking walls. We can consider Facebook and Twitter for this studies and for the safety reason and availability of information, we're thinking about Twitter for this studies. Twitter tweets and tagging, kind of posts they're developing and every now and then a few account human beings creates mess With inside the society with their posts, become aware of the ones and block the fake and undesirable statistics circulating over the community for the peace and safety. Twitter is used as fundamental primarily based totally with ML NLP for the processing of the textual content information and right here we should use sentiment evaluation for figuring out the dreams we placed on.

2.In this paper creator provided the take a look at of diverse techniques for detection of fake profiles. [2] In this paper a take a look at of diverse papersis done, and with inside the reviewed paper we give an explanation for the set of rules and techniques for detecting fake profiles for safety reason. The fundamental a part of this paper covers the safety evaluation of safety on social networking websites. On- line Social Networks (OSNs) are more and more more influencing the manner human beings speak with every different and percentage non-public, expert and political statistics. Increasing reviews of the safety and privateness threats with inside the OSNs is attracting safety researchers attempting to stumble on and mitigate threats to person customers. With many OSNs having tens or loads of million customers together producing billions of non-public information content material that may be exploited, detecting and stopping assaults on person consumer privateness is a predominant challenge. Most of the cutting-edge studies has targeted on shielding the privateness of an existing on-line profile in a given OSN. The fake profile may be exploited to construct on-line dating with the pals of sufferer of identification theft, with the final goal of stealing non-public statistics of the sufferer, through interacting on-line with the pals of the suffer. In this paper, we file at the investigation we did on a probable technique to mitigate this problem.

3.In this paper creator proposed the Social networks have turn out to be an ordinary device in our lives and unique social networks have unique goal groups. [3] Among them LinkedIn is greatly favored via way of means of the individuals who are with inside the expert occupations. With the speedy boom of social networks, human beings have a tendency to misuse them for unethical and unlawful conducts. Creation of a fake profile turns into such adversary impact that's hard to become aware of with out apt studies. The cutting-edge solutions which have been nearly evolved and theorized to remedy this contention, in general taken into consideration the traits and the 
social community ties of the consumer's social profile. However, whilst it comes to LinkedIn such behavioural observations are highly restrictive in publicly to be had profile information for the customers via way of means of the privateness policies. The confined publicly to be had profile information of LinkedIn makes it ineligible in making use of the present strategies in Fake profile identity. Therefore, there may be a want to behavior centered studies on figuring out strategies for fake profile identity in LinkedIn. In this studies, we become aware of the minimal set of profile information which might be important for figuring out Fake profiles in LinkedIn and become aware of the suitable information mining technique for such task. We reveal that with confined profile information our technique can become aware of the fake profile with $84 \%$ accuracy and most effective, that's similar to the effects acquired via way of means of different existing strategies primarily based totally on the bigger information set and more profile statistics.

4.In this paper creator states that the Latest traits have visible exponential boom in clients of social networks.[4] Facebook has 1.5 billion customers. More than 10 million likes and shares are finished daily. Many different networks like ' linkedin, 'Instagram, 'Pinterest, 'Twitter and many others are rapid growing. Growth of social networks has given upward push to a completely excessive quantity of fake consumer profiles created out of ulterior motives. Fake profiles are additionally referred to as Sybils or social Bots. Many such profiles attempt to befriend the benign customers with an final intention of having access to privileged statistics. Social engineering is the primary reason of threats in any Online Social Network(OSN). This paper evaluations many techniques to stumble on the fake profiles and their on-line social bot. Multi agent angle of on-line social networks has additionally been analysed. It additionally discusses the Machine studying techniques beneficial in profile introduction and evaluation.

5.In this paper creator proposed the Social networking websites along with Twitter and Facebook draws tens of thousands and thousands of customers the world over and their interplay with social networking has affected their life. [5]This reputation in social networking has brought about unique issues consisting of the opportunity of disclosing wrong statistics to their customers via fake money owed which ends to the unfold of malicious content material. This state of affairs can end result to a big harm with inside the actual international to the society. In our take a look at, we gift a classification approach for detecting the fake money owed on Twitter. We have pre-processed our dataset the usage of a supervised discretization method named Entropy Minimization Discretization (EMD) on numerical functions and analysed the effects of the Naïve Bayes set of rules.

\section{CONCLUSION}

Fake bills are risky for social systems on the grounds that they may also adjust principles like reputation and have an impact on on Instagram and effect the economy, politics, and society. This paper has brought a fake account detection technique primarily based totally on machine getting to know for the Instagram platform. To attain the proposed techniques goal, we've got created a dataset of valid and fake bills for the Instagram platform. Then, diverse proposals for detecting fake bills had been surveyed primarily based totally on category algorithms and characteristic sets. The brought technique taken into consideration the customers content material and conduct features and carried out them to the bagging classifier set of rules for fake and valid bills category. 


\section{REFERENCES}

[1]. Guo, G., Zhu, Y., Yu, R., Chu, W.C.C., Ma, D. (2020). A privacy-preserving framework with self- governance and permission delegation in online social networks.IEEE Access,8:157116157129.

https://doi.org/10.1109/ACCESS.2020.3016041

[2]. Boididou, C., Middleton, S.E., Jin, Z., Papadopoulos, S., Dang-Nguyen, D.T., Boato, G., Kompatsiaris, Y. (2018). Verifying information with multimedia content on twitter. Multimedia Tools and Applications, 77(12): 15545-15571. https://doi.org/10.1007/s11042-017-5132-9

[3]. Alqatawna, J., Madain, A., Al-Zoubi, A., AlSayyed,R. (2017). Online social networks security: Threats, attacks, and future directions. In Social Media Shaping ePublishing and Academia,pp.121132.

https://doi.org/10.1007/978-3-319-55354-210

[4]. Lorincz, L., Koltai, J., Győr, A.F., Takacs, K. (2019). Collapse of an online social network: Burning social capital to create it? Social Networks,57:43-53.

https://doi.org/10.1016/j.socnet.2018.11.004

[5]. Arora, A., Bansal, S., Kandpal, C., Aswani, R., Dwivedi, Y. (2019). Measuring social media influencer index-insights from Facebook, Twitter and Instagram. Journal Of Retailing and Consumer Services, 49: 86-101. https://doi.org/10.1016/j.jretconser.2019.03.012

[6]. Boerman, S.C. (2020). The effects of the standardized Instagram disclosure for microand meso- influencers. Computers in Human Behavior, 103:199-207. https://doi.org/10.1016/j.chb.2019.09.015

[7]. Yang, C., Harkreader, R., Gu, G. (2013). Empirical evaluation and new design for fighting evolving twitter spammers. IEEE Transactions on Information Forensics and
Security,8(8):1280-1293.

https://doi.org/10.1109/TIFS.2013.2267732

[8]. Han, Y., Fang, B., Jia, Y. (2014). Predicting the topic influence trends in social media with multiple models.Neurocomputing,144:463-470. https://doi.org/10.1016/j.neucom.2014.03.054

[9]. Jr Barbon, S., Igawa, R.A., Zarpelao, B.B. (2017). Authorship verification applied to detection of compromised accounts on online social networks. Multimedia Tools and Applications, 76(3):3213-3233.

https://doi.org/10.1007/s11042-016-3899-8

[10]. Blair, S.J., Bi, Y., Mulvenna, M.D. (2020). Aggregated topic models for increasing social media topic coherence. Applied Intelligence, 50(1): 138-156. https://doi.org/10.1007/s10489019-01438-z

\section{Cite this article as :}

Er. Pranay Meshram, Rutika Bhambulkar, Puja Pokale, Komal Kharbikar, Anushree Awachat, "Survey Paper on Automatic Detection of Fake Profile Using Machine Learning on Instagram", International Journal of Scientific Research in Science, Engineering and Technology (IJSRSET), Online ISSN : 2394-4099, Print ISSN : 2395-1990, Volume 8 Issue 3, pp. 46-50, May-June 2021. Available at doi : https://doi.org/10.32628/IJSRSET218313 Journal URL : https://ijsrset.com/IJSRSET218313 